\title{
LOCAL MAXIMAL OPERATORS ON MEASURE METRIC SPACES
}

\author{
Chin-Cheng Lin, Krzysztof Stempak, and Ya-Shu Wang
}

\begin{abstract}
The notion of local maximal operators and objects associated to them is introduced and systematically studied in the general setting of measure metric spaces. The locality means here some restrictions on the radii of involved balls. The notion encompasses different definitions dispersed throughout the literature. One of the aims of the paper is to compare properties of the 'local' objects with the 'global' ones (i.e. these with no restrictions on the radii of balls). An emphasis is put on the case of locality function of Whitney type. Some aspects of this specific case were investigated earlier by two out of three authors of the present paper.
\end{abstract}

2010 Mathematics Subject Classification: 42B25, 51F99.

Key words: Local maximal operators, measure metric spaces, locality functions of Whitney type, local $A_{p}$ weights, local $B M O$ spaces.

\section{Introduction}

Let $(X, d, \mu)$ be a measure metric space, where $d$ is a distance and $\mu$ is a nonnegative Borel measure on $X$ such that $0<\mu(B(x, r))<\infty$ for all open balls

$$
B(x, r)=\{y \in X: d(x, y)<r\}, \quad x \in X, \quad 0<r<\infty .
$$

Given a lower semicontinuous function $\rho: X \rightarrow(0, \infty]$ (l.s.c. for short), let $\mathcal{O}_{\rho}(x)=\mathcal{O}_{\rho, d}(x)$ denote the family of balls centered at $x$ and with radius $r<\rho(x)$. Then we set

$$
\mathcal{O}_{\rho}=\mathcal{O}_{\rho, d}=\bigcup_{x \in X} \mathcal{O}_{\rho}(x) .
$$

By a local integrability of a complex-valued function on $X$ we mean its integrability with respect to the family of balls from $\mathcal{O}_{\rho}$; thus $f \in$ $L_{\text {loc }, \rho}^{1}(X):=L_{\text {loc }, \rho}^{1}(X, d, \mu)$ provided $\int_{B}|f| d \mu<\infty$ for every ball $B \in \mathcal{O}_{\rho}$.

Research of the first and the third authors supported by NSC of Taiwan under Grant \#NSC 97-2115-M-008-021-MY3. Research of the second author supported by MNiSW under Grant \#N N201 417839. 
(Note that this notion of local integrability does not refer to compactness.)

We define the maximal operator $M_{\rho}$ acting on $f \in L_{\mathrm{loc}, \rho}^{1}(X)$ by

$$
M_{\rho} f(x)=\sup _{x \in B \in \mathcal{O}_{\rho}} \frac{1}{\mu(B)} \int_{B}|f| d \mu, \quad x \in X,
$$

where the supremum is taken over all balls from $\mathcal{O}_{\rho}$ that contain $x$; its centered version is defined by

$$
M_{\rho}^{c} f(x)=\sup _{0<r<\rho(x)} \frac{1}{\mu(B(x, r))} \int_{B(x, r)}|f| d \mu, \quad x \in X .
$$

If $\rho \equiv \infty$ identically, then $M_{\infty}$ and $M_{\infty}^{c}$ are the usual (non-centered and centered) Hardy-Littlewood maximal operators on $(X, d, \mu)$ and $L_{\text {loc }, \infty}^{1}(X)$ is the space of locally integrable (with respect to all balls) functions on $X$; we then skip the $\infty$ subscript and simply write $M, M^{c}$ and $L_{\mathrm{loc}}^{1}(X)$, and refer to this setting as to the global one. Clearly

$$
M_{\rho}^{c} f \leq M_{\rho} f, \quad M_{\rho}^{c} f \leq M^{c} f, \quad M_{\rho} f \leq M f, \quad f \in L_{\mathrm{loc}}^{1}(X) .
$$

The relation $M_{\rho}^{c} \leq M_{\rho}$ shows that some of the (upper) bounds or claims for $M_{\rho}$ imply the corresponding bounds or claims for $M_{\rho}^{c}$ and vice versa (for lower bounds). Usually we shall concentrate on proving bounds or claims for one of the two operators, $M_{\rho}^{c}$ or $M_{\rho}$, this one for which the claim or bound is more subtle.

If $\rho(x) \neq \infty$ for some $x \in X$, then we will refer to $\rho$ as to a locality function and to $M_{\rho}$ and $M_{\rho}^{c}$ as to local Hardy-Littlewood maximal operators. It is clear that in case $\mu$ is doubling, one has $M^{c} f(x) \simeq M f(x)$ uniformly in $f \in L_{\mathrm{loc}}^{1}(X)$ and $x \in X$ (without the doubling condition it may happen that $M^{c}$ and $M$ are not pointwise equivalent). However, in general, even if $\mu$ is doubling, $M_{\rho}^{c} f \simeq M_{\rho} f$ does not hold uniformly in $f \in L_{\text {loc, } \rho}^{1}(X)$; see Example 5.2.

There is a number of papers in the literature where, in different settings, local maximal operators are discussed, sometimes implicitly. For instance, if $X=\mathbb{R}^{n}$ is considered with the Euclidean distance and Lebesgue measure, and $\rho \equiv 1$ (or, more generally, $\rho \equiv a, a>0$ ), then the corresponding local maximal operator was discussed by Rychkov [19]. Ionescu [10] defined and studied the local maximal operator $M_{\rho}$ with $\rho \equiv b, b>0$ being a parameter, in the setting of noncompact symmetric spaces of real rank one (with the canonical distance and measure). Mauceri and Meda [16] used the local Hardy-Littlewood maximal operator $M_{\rho}$ in the context of the measure metric space $\left(\mathbb{R}^{n}, d_{2}, \gamma_{n}\right)$, where $d_{2}$ is the Euclidean distance induced by the norm $\|\cdot\|_{2}$, and 
$\gamma_{n}$ is the Gauss (non-doubling) measure on $\mathbb{R}^{n}$; the corresponding locality function was chosen to be $\rho=\rho_{a}, \rho_{a}(x)=a \min \left(1,1 /\|x\|_{2}\right)$ with parameter $a>0$. In [13] C.-C. Lin and Stempak discussed the local Hardy-Littlewood maximal operator $M_{\rho}$ in the context of $X=\mathbb{R}^{n} \backslash\{0\}$ with Lebesgue measure and the distance $d_{\infty}$ induced by the norm $\|\cdot\|_{\infty}$, where $\rho(x)=k\|x\|_{\infty}, k \in(0,1)$ being a parameter.

In this paper we define the notion of local maximal operators (HardyLittlewood and sharp maximal operators) as well as some associated notions in full generality of measure metric spaces; we then try to keep this generality as far as possible. In particular, we do not assume the doubling condition as a standing assumption. However, it is evident that in order to get a richer theory it is necessary to narrow the setting and assume some additional conditions of geometrical nature on the considered measure metric space. This approach is well represented in the papers by Carbonaro, Mauceri and Meda [4], [5], where, for instance, approximate midpoint or isoperimetric properties were imposed to get satisfactory results. Having defined the local objects we then investigate them from the point of similarities or differences with the global ones. The difference in behavior of local versus global objects is explained by a lack of large balls when $\rho$ is a genuine locality function; this will be seen in several places. Throughout the paper an emphasis is put on the independence of the considered objects of a metric. Note that for $X=\mathbb{R}^{n}$ (or for a subset of $X=\mathbb{R}^{n}$ ), if $d$ is either the Euclidean metric $d_{2}$ or the metric $d_{\infty}$, then geometrically the choice of $d$ results in dealing with Euclidean balls or cubes with sides parallel to the coordinate axes, respectively. In the general case we are a bit more general by considering a pair of arbitrary but equivalent metrics.

The paper is organized as follows. In Section 2 we prove basic facts about local Hardy-Littlewood maximal operators and collect remarks on possible relations between these operators for different locality functions and different metrics. In Section 3 we discuss local sharp maximal operators and local $B M O$ spaces. Section 4 is devoted to a discussion of local $A_{p, \rho}$ weights. Finally, in Section 5 we concentrate the attention on locality function of Whitney type. Numerous examples dispersed within the paper illustrate relations between discussed objects when either a locality function is kept fixed but a metric is replaced onto an equivalent one, or an analogous situation occurs but with the role of a locality function and a metric switched. Notice that the proofs of (almost) all results proved in the paper contain $\rho \equiv \infty$ as a special case.

Throughout the paper we use a standard notation. Writing estimates we use the notation $S \lesssim T$ to indicate that $S \leq C T$ with a positive 
constant $C$ independent of significant quantities. We shall write $S \simeq T$ when simultaneously $S \lesssim T$ and $T \lesssim S$. Given a function $f$ on $X$ we write $\operatorname{supp}^{o} f:=\{x \in X: f(x) \neq 0\}$ to denote the true support of $f$; clearly supp $f$ is the closure of $\operatorname{supp}^{o} f$ in $X$. By $L^{p}(X)=L^{p}(X, \mu)$, $1 \leq p<\infty$, we shall denote the usual Lebesgue $L^{p}$ space on the measure metric space $(X, \mu)$, and $s(\mathbb{N})$ will stand for the space of all complexvalued sequences on $\mathbb{N}$. Finally, if $B=B(x, r)$ and $\tau>0$, then $\tau B=$ $B(x, \tau r)$.

\section{Local Hardy-Littlewood maximal operators}

Recall that throughout this and the next sections $\mu$ is a Borel measure on $X$ which, for a given metric $d$, is positive and finite on all balls related to $d$. At some places we shall additionally assume that $X$ is locally compact and $\mu$ is a Radon measure. By a Radon measure on a locally compact metric space $X$ we mean a Borel measure that is finite on all compact sets, outer regular on all Borel sets, and inner regular on all open sets; cf. [8, Chapter 7]; when a considered measure is Radon then we automatically assume that $X$ is locally compact. The measure $\mu$ will not change but sometimes we shall consider another metric $d^{\prime}$ equivalent with $d$. Clearly, the topologies generated by $d$ and $d^{\prime}$ coincide and so do the families of Borel sets. In particular, $\mu$ is also positive and finite on all balls related to $d^{\prime}$ and the property of being Radon remains unchanged.

For a function $f \in L_{\text {loc }, \rho}^{1}(X)$ its average in a ball $B=B(x, r) \in \mathcal{O}_{\rho}$ will be denoted by

$$
\langle f\rangle_{B}=\frac{1}{\mu(B)} \int_{B} f d \mu .
$$

We start by collecting some basic facts about local maximal operators.

Lemma 2.1. For every $f \in L_{\mathrm{loc}, \rho}^{1}(X), M_{\rho} f$ and $M_{\rho}^{c} f$ are l.s.c., hence Borel measurable.

Proof: In the 'non-centered' case, given $\lambda>0$ and $f \in L_{\text {loc }, \rho}^{1}(X)$ we write the relevant level set as a sum of open balls,

$$
\left\{x \in X: M_{\rho} f(x)>\lambda\right\}=\bigcup_{(y, r) \in D_{f}(\lambda)} B(y, r),
$$

where $D_{f}(\lambda)=\left\{(y, r) \in X \times(0, \infty): r<\rho(y)\right.$ and $\left.\langle|f|\rangle_{B(y, r)}>\lambda\right\}$ (note that l.s.c. of $\rho$ is not required here). In the centered case we write the level set as a sum of open sets

$\left\{x \in X: M_{\rho}^{c} f(x)>\lambda\right\}=\bigcup_{r>0}\left\{y \in X:\langle|f|\rangle_{B(y, r)}>\lambda\right\} \cap\{y \in X: \rho(y)>r\}$. 
Each intersection on the right hand side is an open set since by assumption $\rho$ is l.s.c. and $X \ni y \mapsto\langle|f|\rangle_{B(y, r)}$ is l.s.c. as well. Indeed, for the latter function, since the limit of an increasing sequence of l.s.c. functions is a l.s.c. function it suffices to consider $f=\chi_{A}, \mu(A)<\infty$. But then $X \ni y \mapsto \frac{\mu(A \cap B(y, r))}{\mu(B(y, r))}$ is l.s.c. as a product of two l.s.c. functions: $X \ni y \mapsto \mu(A \cap B(y, r))$ is l.s.c. by continuity of $\mu$ from above, while $X \ni y \mapsto \frac{1}{\mu(B(y, r))}$ is l.s.c. by continuity of $\mu$ from below.

Note that $\langle|f|\rangle_{B}$, and thus $M_{\rho} f$, makes sense for a class of functions larger than $L_{\text {loc }, \rho}^{1}(X)$. In particular, admissible are Borel measurable complex-valued functions, or Borel measurable functions with values in the extended real number system $\overline{\mathbb{R}}=\mathbb{R} \cup\{ \pm \infty\}$. Note that the conclusion of Lemma 2.1 also holds for such functions.

It is well known that for a measure $\mu$ which is doubling, i.e. satisfying

$$
\mu(B(x, 2 r)) \lesssim \mu(B(x, r))
$$

uniformly in $x \in X$ and $r>0$, the operator $M$ is of weak-type $(1,1)$, that is

$$
\mu(\{x \in X: M f(x)>\lambda\}) \lesssim \frac{1}{\lambda}\|f\|_{L^{1}(X, \mu)},
$$

uniformly in $f \in L^{1}(X)$ and $\lambda>0$. Due to (1.1), the same assertion is valid for $M_{\rho}$ (and $M_{\rho}^{c}$ as well). However, we would like to have at our disposal analogous result with much weaker assumptions imposed on $\mu$.

In the literature the notion of local doubling is also used. A measure $\mu$ is said to be local doubling provided for every $b>0$ there exists $C_{b}>0$ such that

$$
\mu(B(x, 2 r)) \leq C_{b} \mu(B(x, r)), \quad x \in X, \quad 0<r \leq b .
$$

This notion is indeed weaker than (global) doubling only for unbounded spaces; if $(X, d)$ is bounded, i.e. $\sup _{x, y \in X} d(x, y)<\infty$, then local doubling implies doubling.

We shall say that $\mu$ satisfies the $\rho$-local $\tau$-condition, $\tau>1$, provided

$$
\mu(\tau B) \lesssim \mu(B), \quad B \in \mathcal{O}_{\rho} .
$$

Proposition 2.2. If $\mu$ satisfies the $\rho$-local 5-condition, then $M_{\rho}$ is of weak-type $(1,1)$. If $\mu$ is Radon, then the $\rho$-local 3 -condition suffices.

Proof: We shall prove that for $\rho_{R}$ defined by $\rho_{R}(x)=\min \{\rho(x), R\}$, it holds

$$
\mu\left(\left\{x \in X: M_{\rho_{R}} f(x)>\lambda\right\}\right) \leq \frac{C}{\lambda}\|f\|_{L^{1}(X, \mu)},
$$


with $C$ independent of $R \rightarrow \infty$. Then

$$
\mu\left(\left\{x \in X: M_{\rho} f(x)>\lambda\right\}\right) \leq \frac{C}{\lambda}\|f\|_{L^{1}(X, \mu)}, \quad \lambda>0, \quad f \in L^{1}(X, \mu),
$$

follows accordingly. Let $x_{0} \in X$ be a reference point. Then (2.2) reduces to checking that

$$
\mu\left(\left\{x \in X: M_{\rho_{R}} f(x)>\lambda\right\} \cap B\left(x_{0}, R^{\prime}\right)\right) \leq \frac{C}{\lambda}\|f\|_{L^{1}(X, \mu)},
$$

with $C$ independent of $0<R<R^{\prime} \rightarrow \infty$. Given $x \in F\left(R, R^{\prime} ; \lambda\right)=\{x \in$ $\left.X: M_{\rho_{R}} f(x)>\lambda\right\} \cap B\left(x_{0}, R^{\prime}\right)$ take a ball $B_{x}=B\left(x, r_{x}\right)$ such that $0<$ $r_{x}<\min \{\rho(x), R\}$ (in particular, $B_{x} \in \mathcal{O}_{\rho}$ ) and $\frac{1}{\mu\left(B_{x}\right)} \int_{B_{x}}|f| d \mu>\lambda$. By the basic covering theorem, [9, Theorem 1.2], it is possible to extract from the family of balls $\left\{B_{x}\right\}_{x \in F\left(R, R^{\prime} ; \lambda\right)}$ of uniformly bounded radii, a subfamily $\left\{B_{x_{s}}\right\}_{s \in S}$ of disjoint balls such that

$$
\bigcup_{x \in F\left(R, R^{\prime} ; \lambda\right)} B_{x} \subset \bigcup_{s \in S} 5 B_{x_{s}} .
$$

Since $R^{\prime}>R$, for every $s \in S, B_{x_{s}} \subset B\left(x_{0}, 3 R^{\prime}\right)$, and hence the family $\left\{B_{x_{s}}\right\}_{s \in S}$ is at most countable. Therefore,

$$
\begin{aligned}
\mu\left(F\left(R, R^{\prime} ; \lambda\right)\right) & \leq \sum_{s \in S} \mu\left(5 B_{x_{s}}\right) \leq C_{5} \sum_{s \in S} \mu\left(B_{x_{s}}\right) \\
& \leq C_{5} \frac{1}{\lambda} \sum_{s \in S} \int_{B_{x_{s}}}|f| d \mu \leq C_{5} \frac{1}{\lambda}\|f\|_{L^{1}(X, \mu)} .
\end{aligned}
$$

In the case when $\mu$ is Radon the proof is a bit easier. As in the classical case, we take an arbitrary compact subset of the level set $\{x \in$ $\left.X: M_{\rho} f(x)>\lambda\right\}$, cover it properly by a family of balls and choose a finite subcover. The Vitali-Wiener covering lemma allows to choose from a given finite family of balls $\left\{B_{1}, B_{2}, \ldots, B_{m}\right\} \subset \mathcal{O}_{\rho}$, a subfamily of pairwise disjoint balls $\left\{B_{j_{1}}, B_{j_{2}}, \ldots, B_{j_{n}}\right\}$ such that

$$
\bigcup_{i=1}^{m} B_{i} \subset \bigcup_{s=1}^{n} 3 B_{j_{s}}
$$

The fact that $\mu$ is Radon (inner regularity on open sets, to be precise) finishes the argument.

It is worth mentioning that in the case of $\mathbb{R}^{n}$, weak type $(1,1)$ inequality (2.1) holds with $M^{c}$ replacing $M$ without assuming that $\mu$ is doubling (Besicovitch's covering lemma is used in the proof). Note also that in the case of $X=\mathbb{R}$, the estimate (2.1) holds without any special 
assumption on $\mu$. This is a simple consequence of a specific covering lemma valid for subsets of $\mathbb{R}$, see [6, Lemma 2.6].

We shall say that $(X, d, \rho)$ is $\sigma$-finite provided $X=\bigcup_{j=1}^{\infty} B_{j}, B_{j} \in \mathcal{O}_{\rho}$.

Proposition 2.3. Let $(X, d, \rho)$ be $\sigma$-finite and let a Radon measure $\mu$ satisfy the $\rho$-local 3-condition. Then for every $f \in L_{\mathrm{loc}, \rho}^{1}(X)$ we have

$$
f(x)=\lim _{x \in B \in \mathcal{O}_{\rho}}\langle f\rangle_{B}, \quad \mu \text {-a.e. } x \in X,
$$

where the limit is understood as a generalized limit with respect to the net (directed by the set inclusion) of balls from $\mathcal{O}_{\rho}$ that contain $x$; in particular, $f(x)=\lim _{r \rightarrow 0^{+}}\langle f\rangle_{B(x, r)}$, $\mu$-a.e. $x \in X$.

Proof: By the assumption made on $X$, a standard localization argument shows that it is sufficient to consider $f \in L^{1}(X)$. It is also easily seen that $(2.3)$ holds true for functions from $C_{c}(X)$ and the latter space is dense in $L^{1}(X)$ since $\mu$ is Radon. Let

$$
E_{n}=\left\{x \in X: \limsup _{x \in B \in \mathcal{O}_{\rho}}\left|\langle f\rangle_{B}-f(x)\right|>1 / n\right\} .
$$

Given $\epsilon>0$ take $g \in C_{c}(X)$ such that $\|f-g\|_{1}<\epsilon$. Then for any $x \in X$

$$
\limsup _{x \in B \in \mathcal{O}_{\rho}}\left|\langle f\rangle_{B}-f(x)\right| \leq M_{\rho}(f-g)(x)+|g(x)-f(x)|
$$

and hence

$$
\begin{aligned}
\mu\left(E_{n}\right) \leq & \mu\left(\left\{x \in X: M_{\rho}(f-g)(x)>\frac{1}{2 n}\right\}\right) \\
& +\mu\left(\left\{x \in X:|f(x)-g(x)|>\frac{1}{2 n}\right\}\right) \\
\leq & 2 n C\|f-g\|_{1}+2 n\|f-g\|_{1} \\
\leq & 2 n(C+1) \epsilon
\end{aligned}
$$

we used Proposition 2.2 and Chebyshev's inequality. Since $\epsilon$ is arbitrary, $\mu\left(E_{n}\right)=0$ for any $n \in \mathbb{N}$ and (2.3) follows.

In the two remarks that follow we comment on possible relations between local maximal operators for different but equivalent locality functions or metrics.

Remark 2.4. It is obvious that $\rho \leq \rho^{\prime}$ implies $\mathcal{O}_{\rho} \subset \mathcal{O}_{\rho^{\prime}}$ and consequently, $L_{\text {loc }, \rho^{\prime}}^{1}(X) \subset L_{\text {loc }, \rho}^{1}(X)$ and $M_{\rho} f \leq M_{\rho^{\prime}} f$ for $f \in L_{\text {loc }, \rho^{\prime}}^{1}(X)$. However, in general, even though $\rho \simeq \rho^{\prime}$, there is no reason to expect that the spaces $L_{\text {loc }, \rho}^{1}(X)$ and $L_{\text {loc }, \rho^{\prime}}^{1}(X)$ coincide. For instance, take $X=\mathbb{N}, d$ to 
be the $0-1$ metric on $\mathbb{N}$ and $\mu$ the measure on $\mathbb{N}$ such that $\mu(\{n\})=a_{n}$, where $a_{n}>0$ and $\sum a_{n}<\infty$ (so that $\left.\mu(\mathbb{N})<\infty\right)$. Note that $\mu$ is not even local doubling and if $B$ is a ball, then either $B=\{n\}$ for some $n \in \mathbb{N}$ or $B=\mathbb{N}$. With $\rho \equiv 1$ and $\rho^{\prime} \equiv 2, L_{\text {loc }, \rho}^{1}(\mathbb{N})=s(\mathbb{N})$, while $L_{\mathrm{loc}, \rho^{\prime}}^{1}(\mathbb{N})=\ell^{1}(\mathbb{N}, \mu)$. But even if $L_{\mathrm{loc}, \rho}^{1}(X)=L_{\mathrm{loc}, \rho^{\prime}}^{1}(X)$, still there is no reason to expect that

$$
M_{\rho} f \simeq M_{\rho^{\prime}} f
$$

uniformly in $f \in L_{\text {loc }, \rho}^{1}(X)=L_{\text {loc, }, \rho^{\prime}}^{1}(X)$. See Example 5.3. Analogous remarks apply to the centered local maximal operators.

Remark 2.5. In general, if $d^{\prime}$ is a metric equivalent with $d, d^{\prime} \simeq d$, there is no reason for the spaces $L_{\mathrm{loc}, \rho, d}^{1}(X)$ and $L_{\mathrm{loc}, \rho, d^{\prime}}^{1}(X)$ to coincide (additional subscripts indicate the dependence of the local $L^{1}$ space on the metric; this agreement will be also used in similar occurrences). For instance, let $X, d, \mu$ and $\rho$ be as in Remark 2.4. Define $d^{\prime}=$ $\frac{1}{2} d$. Then $L_{\text {loc }, \rho, d}^{1}(\mathbb{N})=s(\mathbb{N})$, while $L_{\text {loc }, \rho, d^{\prime}}^{1}(\mathbb{N})=\ell^{1}(\mathbb{N}, \mu)$. But even if $L_{\mathrm{loc}, \rho, d}^{1}(X)=L_{\mathrm{loc}, \rho, d^{\prime}}^{1}(X)$, then still there is no reason for the equivalence

$$
M_{\rho, d}^{c} f \simeq M_{\rho, d^{\prime}}^{c} f
$$

to hold, uniformly in $f \in L_{\mathrm{loc}, \rho, d}^{1}(X)=L_{\mathrm{loc}, \rho, d^{\prime}}^{1}(X)$. See Example 5.4. Analogous remarks apply to non-centered local maximal operators.

Finally, the next two remarks concern the local $L^{1}$ spaces.

Remark 2.6. Given two different l.s.c. functions $\rho^{\prime}$ and $\rho$ on $(X, d, \mu)$ assume that every ball from $\mathcal{O}_{\rho^{\prime}}$ may be covered by a finite number of balls from $\mathcal{O}_{\rho}$ and vice versa with the role of $\rho^{\prime}$ and $\rho$ exchanged. Then

$$
L_{\mathrm{loc}, \rho^{\prime}}^{1}(X)=L_{\mathrm{loc}, \rho}^{1}(X) .
$$

In concrete settings it frequently happens (and is supported by numerous examples) that for $\rho^{\prime}$ and $\rho$ as above, the discussed covering property holds.

Remark 2.7. Similarly, if $\rho$ is fixed, given two different metrics $d^{\prime}$ and $d$ such that every ball from $\mathcal{O}_{\rho, d^{\prime}}$ may be covered by a finite number of balls from $\mathcal{O}_{\rho, d}$ and vice versa with the role of $d^{\prime}$ and $d$ exchanged, then

$$
L_{\mathrm{loc}, \rho, d}^{1}(X)=L_{\mathrm{loc}, \rho, d^{\prime}}^{1}(X) .
$$

Again a similar comment as in Remark 2.6 applies. 


\section{Local sharp maximal operators and local $B M O$ spaces}

In this section we introduce the notion of local sharp maximal operators and local $B M O$ spaces. We then study their basic properties, investigate relations between local $B M O$ spaces for different metrics and furnish some examples.

Let the system $(X, d, \mu, \rho)$ be given. Keeping the notation from Section 2 we define the sharp maximal operator $M_{\rho}^{\#}$ acting on $f \in L_{\text {loc, } \rho}^{1}(X)$ by

$$
M_{\rho}^{\#} f(x)=\sup _{x \in B \in \mathcal{O}_{\rho}} \frac{1}{\mu(B)} \int_{B}\left|f-\langle f\rangle_{B}\right| d \mu, \quad x \in X,
$$

where the supremum is taken over all balls from $\mathcal{O}_{\rho}$ that contain $x$; its centered version is given by

$$
M_{\rho}^{\#, c} f(x)=\sup _{0<r<\rho(x)} \frac{1}{\mu(B(x, r))} \int_{B(x, r)}\left|f-\langle f\rangle_{B(x, r)}\right| d \mu, \quad x \in X .
$$

If $\rho \equiv \infty$, then $M_{\infty}^{\#}$ and $M_{\infty}^{\#, c}$ are the usual (non-centered and centered, both appear in the literature) sharp maximal operators on $(X, d, \mu)$; we then skip the $\infty$ subscript and simply write $M^{\#}$ and $M^{\#, c}$. If $\rho$ is a locality function, then we shall refer to $M_{\rho}^{\#}$ and $M_{\rho}^{\#, c}$ as to local sharp maximal operators. Clearly

$$
M_{\rho}^{\#, c} f \leq M_{\rho}^{\#} f, \quad M_{\rho}^{\#, c} f \leq M^{\#, c} f, \quad M_{\rho}^{\#} f \leq M^{\#} f, \quad f \in L_{\mathrm{loc}, \rho}^{1}(X) .
$$

The space $B M O_{\rho}(X):=B M O_{\rho}(X, d, \mu)$ is defined to consist of all $f \in L_{\mathrm{loc}, \rho}^{1}(X)$ for which

$$
\|f\|_{*, \rho}:=\sup _{x \in X} M_{\rho}^{\#, c} f(x)<\infty .
$$

Note that the pointwise equivalence $M_{\rho}^{\#, c} f(x) \simeq M_{\rho}^{\#} f(x)$ may not hold uniformly in $f \in L_{\mathrm{loc}, \rho}^{1}(X)$ and $x \in X$, however the identity

$$
\sup _{x \in X} M_{\rho}^{\#, c} f(x)=\sup _{x \in X} M_{\rho}^{\#} f(x)
$$

holds for any $f \in L_{\text {loc }, \rho}^{1}(X)$ and, moreover,

$$
\|f\|_{*, \rho}=\sup _{B \in \mathcal{O}_{\rho}} \frac{1}{\mu(B)} \int_{B}\left|f-\langle f\rangle_{B}\right| \simeq \sup _{B \in \mathcal{O}_{\rho}} \inf _{c \in \mathbb{C}} \frac{1}{\mu(B)} \int_{B}|f-c| .
$$

If $\rho$ is a locality function, then we will refer to $B M O_{\rho}(X)$ as to a local $B M O$ space. If $\rho \equiv \infty$, then $B M O_{\infty}(X)$ becomes the usual $B M O$ space on the measure metric space $(X, d, \mu)$ and is denoted as $B M O(X)$. 
In recent years $B M O$ spaces with 'local flavour' were defined and investigated in several settings, some of them fitting into the scheme presented above. Ionescu [10] defined and studied the local sharp maximal operator $M_{\rho}^{\#}$ with $\rho \equiv 1$ in the setting of noncompact symmetric spaces of real rank one. Mauceri and Meda [16] defined and studied the space $B M O_{\rho_{a}}\left(\mathbb{R}^{n}, d, \gamma_{n}\right)$, where $d, \gamma_{n}$ and $\rho_{a}$ are as described earlier. Later on Carbonaro, Mauceri and Meda [4], [5], extended ideas from [10] and [16] to a general context of measure metric spaces with measure satisfying local doubling property (and some additional geometric properties as well) and $\rho=\rho_{b}$ chosen to be $\rho \equiv b, b>0$ being a parameter. To be precise in [5], [16] (the case of $\mu(X)<\infty$ ), in the definition of the local $B M O$ space it was assumed that $f \in L^{1}\left(\mathbb{R}^{n}, \gamma_{n}\right)$ rather than a weaker condition $f \in L_{\text {loc }, \rho}^{1}\left(\mathbb{R}^{n}, \gamma_{n}\right)$. Similarly, in [4] (the case of $\mu(X)=\infty$ ), it was assumed that $f \in L_{\text {loc }}^{1}(X, \mu)$, which is again a stronger condition than $f \in L_{\text {loc }, \rho}^{1}(X, \mu)$ for $\rho \not \equiv \infty$. Recently, Lin and Stempak [14] defined and investigated the spaces $B M O_{\rho}(\Omega)$, where $\Omega$ and $\rho=\rho_{k}$ are as in Section 5. It is interesting to point out that in the case of the upper half-space $\mathbb{R}_{+}^{n}=\left\{\left(x_{1}, \ldots, x_{n}\right) \in \mathbb{R}^{n}: x_{n}>0\right\}$ or the unit ball $B(0,1) \subset \mathbb{R}^{n}$, this type of $B M O$ space was considered by Reimann and Rychener [18, p. 4]; see also [7, Remark 2.3].

Lemma 3.1. For every $f \in L_{\mathrm{loc}, \rho}^{1}(X), M_{\rho}^{\#} f$ and $M_{\rho}^{\#, c} f$ are l.s.c., hence Borel measurable.

Proof: As in the proof of Lemma 2.1 we write

$$
\left\{x \in X: M_{\rho}^{\#} f(x)>\lambda\right\}=\bigcup_{(y, r) \in E_{f}(\lambda)} B(y, r),
$$

where $E_{f}(\lambda)=\left\{(y, r) \in X \times(0, \infty): r<\rho(y)\right.$ and $\left.\left\langle\left|f-\langle f\rangle_{B(y, r)}\right|\right\rangle_{B(y, r)}\right\rangle$ $\lambda\}$. In the centered case we write the level set as a sum of open sets

$$
\begin{array}{r}
\left\{x \in X: M_{\rho}^{\#, c} f(x)>\lambda\right\}=\bigcup_{r>0}\left\{y \in X:\left\langle\left|f-\langle f\rangle_{B(y, r)}\right|\right\rangle_{B(y, r)}>\lambda\right\} \\
\cap\{y \in X: \rho(y)>r\} .
\end{array}
$$

As in the proof of Lemma 2.1 checking that the first set in the intersection is open requires verifying that the relevant function is l.s.c. This reduces to considering $f=\chi_{A}, \mu(A)<\infty$, and thus the function $X \ni y \mapsto$ $\left(1-\frac{\mu(A \cap B(y, r))}{\mu(B(y, r))}\right) \frac{\mu(A \cap B(y, r))}{\mu(B(y, r))}$, which is l.s.c. by the argument already used in Lemma 2.1 .

It may happen that $B M O_{\rho}(X)=L_{\text {loc }, \rho}^{1}(X)$ and $\|f\|_{*, \rho}=0$ for every $f \in B M O_{\rho}(X)$ (for instance, take $X=\mathbb{N}$ with usual distance and 
counting measure, and $\rho \equiv 1)$. To avoid such trivialities we assume that the system $(X, d, \mu, \rho)$ is such that $\|f\|_{*, \rho}>0$ for some $f \in B M O_{\rho}(X)$. Also, since $\|\cdot\|_{*, \rho}$ is only a seminorm, to generate a genuine norm in the quotient space one has to determine the subspace

$$
W_{0, \rho}=\left\{f \in B M O_{\rho}(X):\|f\|_{*, \rho}=0\right\} .
$$

Unlike to the case of $\rho \equiv \infty, W_{0, \rho}$ may be bigger than the space of constant functions. For instance, consider again $\mathbb{N}$ with usual distance and counting measure, and $\rho$ given by: $\rho(n)=1$ for $1 \leq n \leq 9$ and $\rho(n)=2$ for $n \geq 10$. Then $W_{0, \rho}$ consists of all sequences $\{f(n)\}$ which are constant for $n \geq 9$.

To determine the shape of $W_{0, \rho}$ we introduce the following notion. Given $x, y \in X$ we write $x \sim_{\rho} y$ provided there exist balls $\left\{B_{1}, \ldots, B_{m}\right\} \subset$ $\mathcal{O}_{\rho}$ such that $x \in B_{1}, y \in B_{m}$ and $B_{i} \cap B_{i+1} \neq \varnothing, i=1, \ldots, m-1$. Clearly, $U_{x}=\left\{y \in X: x \sim_{\rho} y\right\}$ is an open set which we call the $\rho$-component of $x$. It is also clear that any two $\rho$-components either coincide or are disjoint and for $\rho \equiv \infty$, the whole space is the only $\rho$-component of this space. Thus $X$ splits into pairwise disjoint open $\rho$-components. It is easy to observe that $W_{0, \rho}$ is the space of functions which are constant $\mu$-a.e. on each of $\rho$-components of $X$.

Proposition 3.2. The quotient space $B M O_{\rho}(X) / W_{0, \rho}$ is a Banach space under the norm $\|\cdot\|_{*, \rho}$.

Proof: Only proving completeness is interesting. Since $B M O_{\rho}(X) / W_{0, \rho}$ is a direct sum of $B M O$ spaces modulo constants on $\rho$-components of $X$, therefore we can assume that there is only one $\rho$-component, namely $X$. Consider a sequence of cosets $\left\{\left[f_{j}\right]\right\}_{j \in \mathbb{N}}$ in $B M O_{\rho}(X) / W_{0, \rho}$ such that

$$
\sum_{j}\left\|\left[f_{j}\right]\right\|_{*, \rho}<\infty
$$

Choosing a reference ball $B_{0} \in \mathcal{O}_{\rho}$ we may assume that $\left\langle f_{j}\right\rangle_{B_{0}}=0$ for every $j$. Then it is rather routine to justify that $\sum f_{j}$ converges in $L^{1}(B)$, and hence $\mu$-a.e. on $B$, for every ball $B \in \mathcal{O}_{\rho}$. Therefore $\sum f_{j}$ is well defined on $X$ and $\langle f\rangle_{B}=\sum_{j}\left\langle f_{j}\right\rangle_{B}$ for any $B \in \mathcal{O}_{\rho}$. Moreover, $\sum_{j} \in B M O_{\rho},\left\|\left[\sum_{1}^{N} f_{j}\right]-[f]\right\|_{*, \rho} \rightarrow 0$ as $N \rightarrow \infty$, which means that
$\sum_{1}^{\infty}\left[f_{j}\right]$ converges in $B M O_{\rho}(X) / W_{0, \rho}$.

At this point a question arises on a possible relation between the spaces $B M O_{\rho^{\prime}}(X)$ and $B M O_{\rho}(X)$ for a given $(X, d, \mu)$, where $\rho^{\prime}$ and $\rho$ are two different but equivalent locality functions. Even if we assume that $L_{\mathrm{loc}, \rho^{\prime}}^{1}(X)=L_{\mathrm{loc}, \rho}^{1}(X)$, there is no reason to expect that $B M O_{\rho^{\prime}}(X)=$ 
$B M O_{\rho}(X)$, with equivalence of norms, say; see Example 3.6. However, in several settings this occurs to be the case (but in each setting this is a nontrivial fact). Mauceri and Meda [16, Proposition 2.4] showed that for $\left(\mathbb{R}^{n}, d_{2}, \gamma_{n}\right)$, the spaces $B M O_{\rho_{a}}\left(\mathbb{R}^{n}, d_{2}, \gamma_{n}\right), a>0$, coincide with equivalence of norms. Moreover, $d_{2}$ may be replaced by $d_{\infty}$ and the result remains true. Similar result was proved by Carbonaro, Mauceri and Meda [4], [5], in the context of a measure metric space $(X, d, \mu)$ with measure satisfying the local doubling property. Under additional assumption on $X$ it was proved that $B M O_{\rho_{b}}(X, d, \mu)$ coincide with equivalence of norms for some range of the parameter $b>0$. Finally, Lin and Stempak [14, Theorem 4.2] showed that the spaces $B M O_{k}(\Omega, d, \lambda)$, $0<k<1, d=d_{2}$ or $d=d_{\infty}$, coincide with equivalence of norms.

Another issue to be considered is the following. For a given $\rho$ and for two equivalent metrics $d^{\prime}$ and $d$, assume that $L_{\text {loc, }, d^{\prime}}^{1}(X)=L_{\text {loc }, \rho, d}^{1}(X)$. The above-mentioned results show that $B M O_{\rho, d^{\prime}}(X)=B M O_{\rho, d}(X)$ may happen (in nontrivial settings) but in general the identity fails; see a simple Example 3.6. (Mateu et al. [15, Theorems 4,5] showed that the claim is false in the case $\rho \equiv \infty)$.

We now present a result that shows the coincidence of local $B M O$ spaces corresponding to the same $\rho$ but different metrics $d$ and $d^{\prime}$. It is worth noticing that the assumed hypotheses are not too restrictive; in particular a use of an "intermediate" function $\rho^{\prime}$ is supported by some concrete settings.

Proposition 3.3. Let $\rho, \rho^{\prime}$ and $d, d^{\prime}$ be given. Assume that

(i) $L_{\mathrm{loc}, \rho, d}^{1}(X)=L_{\mathrm{loc}, \rho^{\prime}, d}^{1}(X)$ and $L_{\mathrm{loc}, \rho, d^{\prime}}^{1}(X)=L_{\mathrm{loc}, \rho^{\prime}, d^{\prime}}^{1}(X)$;

(ii) $B M O_{\rho, d}(X)=B M O_{\rho^{\prime}, d}(X)$ and $B M O_{\rho, d^{\prime}}(X)=B M O_{\rho^{\prime}, d^{\prime}}(X)$ with equivalence of the corresponding norms;

(iii) for every $B \in \mathcal{O}_{\rho, d}$ there exists $\hat{B} \in \mathcal{O}_{\rho^{\prime}, d^{\prime}}$ such that $B \subset \hat{B}$ and $\mu(\hat{B}) \simeq \mu(B) ;$

(iv) for every $B^{\prime} \in \mathcal{O}_{\rho, d^{\prime}}$ there exists $\check{B}^{\prime} \in \mathcal{O}_{\rho^{\prime}, d}$ such that $B^{\prime} \subset \check{B}^{\prime}$ and $\mu\left(\check{B}^{\prime}\right) \simeq \mu(B)$.

Then $L_{\mathrm{loc}, \rho, d}^{1}(X)=L_{\mathrm{loc}, \rho, d^{\prime}}^{1}(X)$ and

$$
B M O_{\rho, d}(X)=B M O_{\rho, d^{\prime}}(X)
$$

with equivalence of the corresponding norms.

Proof: Checking that the local $L^{1}$ spaces corresponding to $(\rho, d)$ and $\left(\rho, d^{\prime}\right)$ coincide is routine by using (iii), (iv) and (i). To prove (3.1) take $f \in L_{\text {loc }, \rho, d}^{1}(X)=L_{\text {loc }, \rho, d^{\prime}}^{1}(X)$ and start with the inclusion $\subset$. For any 
$B \in \mathcal{O}_{\rho, d}$ take $\hat{B} \in \mathcal{O}_{\rho^{\prime}, d^{\prime}}$ as in (iii). Then

$$
\begin{aligned}
\inf _{c \in \mathbb{C}} \frac{1}{\mu(B)} \int_{B}|f-c| d \mu & \lesssim \inf _{c \in \mathbb{C}} \frac{1}{\mu(\hat{B})} \int_{\hat{B}}|f-c| d \mu \\
& \leq \sup _{B^{\prime} \in \mathcal{O}_{\rho^{\prime}, d^{\prime}}} \inf _{c \in \mathbb{C}} \frac{1}{\mu\left(B^{\prime}\right)} \int_{B^{\prime}}|f-c| d \mu \\
& \simeq\|f\|_{*, \rho^{\prime}, d^{\prime}}
\end{aligned}
$$

Thus

$$
\|f\|_{*, \rho, d} \lesssim\|f\|_{*, \rho^{\prime}, d^{\prime}} \lesssim\|f\|_{*, \rho, d^{\prime}}
$$

To prove the opposite inclusion, given $B^{\prime} \in \mathcal{O}_{\rho, d^{\prime}}$, take $\check{B}^{\prime} \in \mathcal{O}_{\rho^{\prime}, d}$ as in (iv). Then

$$
\begin{aligned}
\inf _{c \in \mathbb{C}} \frac{1}{\mu\left(B^{\prime}\right)} \int_{B^{\prime}}|f-c| d \mu & \lesssim \inf _{c \in \mathbb{C}} \frac{1}{\mu\left(\check{B}^{\prime}\right)} \int_{\check{B}^{\prime}}|f-c| d \mu \\
& \leq \sup _{B \in \mathcal{O}_{\rho^{\prime}, d}} \inf _{c \in \mathbb{C}} \frac{1}{\mu(B)} \int_{B}|f-c| d \mu \\
& \simeq\|f\|_{*, \rho^{\prime}, d}
\end{aligned}
$$

Thus

$$
\|f\|_{*, \rho, d^{\prime}} \lesssim\|f\|_{*, \rho^{\prime}, d} \lesssim\|f\|_{*, \rho, d}
$$

The result that follows is well known in the 'global' setting (the case of $\rho \equiv \infty)$ and provides an equivalent statement for the John-Nirenberg inequality (3.2). We furnish the proof of the local version, which is based on a known argument, for the sake of completeness.

Lemma 3.4. The following two statements are equivalent.

(1) there exist $C_{1}, C_{2}>0$ such that for every $f \in B M O_{\rho}(X)$ and every $B \in \mathcal{O}_{\rho}$

$$
\mu\left(\left\{x \in B:\left|f(x)-\langle f\rangle_{B}\right|>\lambda\right\}\right) \leq C_{1} \exp \left(-\frac{C_{2}}{\|f\|_{*, \rho}} \lambda\right) \mu(B), \quad \lambda>0
$$

(2) there exist $C_{3}, C_{4}>0$ such that for every $f \in B M O_{\rho}(X)$ and every $B \in \mathcal{O}_{\rho}$

$$
\int_{B} \exp \left(\frac{C_{3}}{\|f\|_{*, \rho}}\left|f-\langle f\rangle_{B}\right|\right) d \mu \leq C_{4} \mu(B) .
$$


Proof: $(1) \Rightarrow(2)$ : For any $p \in[1, \infty)$ and $B \in \mathcal{O}_{\rho},(3.2)$ implies (see, for instance, $[\mathbf{6}$, p. 125$])$

$$
\int_{B}\left|f-\langle f\rangle_{B}\right|^{p} d \mu \leq \frac{C_{1}}{C_{2}^{p}} \Gamma(p+1)\|f\|_{*, \rho}^{p} \mu(B) .
$$

Thus, expanding $\exp \left(\frac{C_{2}}{2\|f\|_{*, \rho}}\left|f-\langle f\rangle_{B}\right|\right)$ into a series and integrating its parts gives

$$
\int_{B} \exp \left(\frac{C_{2}}{2\|f\|_{*, \rho}}\left|f-\langle f\rangle_{B}\right|\right) d \mu=\sum_{j=0}^{\infty} \frac{C_{2}^{j} 2^{-j}}{j !\|f\|_{*, \rho}^{j}} \int_{B}\left|f-\langle f\rangle_{B}\right|^{j} d \mu \leq C_{1} \mu(B) .
$$

Hence (3.3) follows with $C_{3}:=C_{2} / 2$ and $C_{4}:=C_{1}$.

$(2) \Rightarrow(1)$ : Given $f \in B M O_{\rho}(X)$ and $B \in \mathcal{O}_{\rho}$ let

$$
E_{\lambda}=\left\{x \in B:\left|f(x)-\langle f\rangle_{B}\right|>\lambda\right\}, \quad \lambda>0 .
$$

Then

$$
\begin{aligned}
\exp \left(\frac{C_{3} \lambda}{\|f\|_{*, \rho}}\right) \mu\left(E_{\lambda}\right) & =\int_{E_{\lambda}} \exp \left(\frac{C_{3} \lambda}{\|f\|_{*, \rho}}\right) d \mu \\
& \leq \int_{E_{\lambda}} \exp \left(\frac{C_{3}\left|f-\langle f\rangle_{B}\right|}{\|f\|_{*, \rho}}\right) d \mu \\
& \leq \int_{B} \exp \left(\frac{C_{3}\left|f-\langle f\rangle_{B}\right|}{\|f\|_{*, \rho}}\right) d \mu \\
& \leq C_{4} \mu(B) .
\end{aligned}
$$

Hence (3.2) follows with $C_{1}:=C_{4}$ and $C_{2}:=C_{3}$.

We shall say that $(X, d, \mu, \rho)$ possesses the John-Nirenberg property provided any of the two equivalent statements of Lemma 3.4 is satisfied. In the case of doubling measure $\mu$ and $\rho \equiv \infty$ it is well known that the John-Nirenberg inequality holds, see [15] and comments included there. On the other hand, examples of measure metric spaces where JohnNirenberg inequality fails to hold are also known, see [15, Section 2]. At the end of this section we take an opportunity to present a simple example of such case, see Example 3.7.

The last result of this section shows that under some (mild) assumptions the John-Nirenberg property may be transferred from one to another local setting.

Proposition 3.5. Suppose the assumptions of Proposition 3.3 hold. Then the John-Nirenberg property for the system $\left(X, d, \mu, \rho^{\prime}\right)$ implies the John-Nirenberg property for $\left(X, d^{\prime}, \mu, \rho\right)$. 
Proof: We first prove that the inequality

$$
\int_{B} \exp \left(\frac{C_{3}}{\|f\|_{*, \rho^{\prime}, d}}\left|f-\langle f\rangle_{B}\right|\right) d \mu \leq C_{4} \mu(B), \quad B \in \mathcal{O}_{\rho^{\prime}, d},
$$

implies

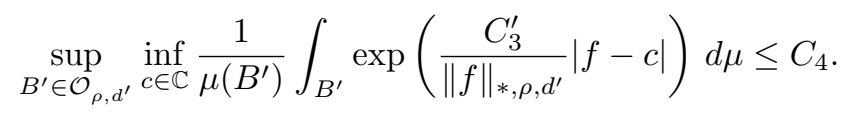

Indeed, specifying the constants in the equivalence in (ii) of Proposition 3.3 ,

$$
\begin{aligned}
& \alpha^{-1}\|\cdot\|_{*, \rho, d^{\prime}} \leq\|\cdot\|_{*, \rho, d} \leq \alpha\|\cdot\|_{*, \rho, d^{\prime}}, \\
& \beta^{-1}\|\cdot\|_{*, \rho, d} \leq\|\cdot\|_{*, \rho^{\prime}, d} \leq \beta\|\cdot\|_{*, \rho, d},
\end{aligned}
$$

take $C_{3}^{\prime}:=\frac{C_{3}}{\alpha \beta}$. Let $B^{\prime} \in \mathcal{O}_{\rho, d^{\prime}}$ and $\check{B}^{\prime} \in \mathcal{O}_{\rho^{\prime}, d}$ be as in (iv) of Proposition 3.3. Then for $c \in \mathbb{C}$,

$$
\begin{aligned}
\frac{1}{\mu\left(B^{\prime}\right)} \int_{B^{\prime}} \exp \left(\frac{C_{3}^{\prime}}{\|f\|_{*, \rho, d^{\prime}}}|f-c|\right) d \mu & \leq \frac{1}{\mu\left(B^{\prime}\right)} \int_{B^{\prime}} \exp \left(\frac{C_{3}^{\prime} \alpha}{\|f\|_{*, \rho, d}}|f-c|\right) d \mu \\
& \lesssim \frac{1}{\mu\left(\check{B}^{\prime}\right)} \int_{\check{B}^{\prime}} \exp \left(\frac{C_{3}^{\prime} \alpha}{\|f\|_{*, \rho, d}}|f-c|\right) d \mu \\
& \leq \frac{1}{\mu\left(\check{B}^{\prime}\right)} \int_{\check{B}^{\prime}} \exp \left(\frac{C_{3}^{\prime} \alpha \beta}{\|f\|_{*, \rho^{\prime}, d}}|f-c|\right) d \mu
\end{aligned}
$$

Hence

$$
\begin{aligned}
\inf _{c \in \mathbb{C}} & \frac{1}{\mu\left(B^{\prime}\right)} \int_{B^{\prime}} \exp \left(\frac{C_{3}^{\prime}}{\|f\|_{*, \rho, d^{\prime}}}|f-c|\right) d \mu \\
& \leq \inf _{c \in \mathbb{C}} \frac{1}{\mu\left(\check{B}^{\prime}\right)} \int_{\check{B}^{\prime}} \exp \left(\frac{C_{3}}{\|f\|_{*, \rho^{\prime}, d}}|f-c|\right) d \mu \\
& \leq \frac{1}{\mu\left(\check{B}^{\prime}\right)} \int_{\check{B}^{\prime}} \exp \left(\frac{C_{3}}{\|f\|_{*, \rho^{\prime}, d}}\left|f-\langle f\rangle_{B^{\prime}}\right|\right) d \mu \\
& \leq C_{4}
\end{aligned}
$$

and (3.4) follows. Finally we show that (3.4) implies

$$
\sup _{B^{\prime} \in \mathcal{O}_{\rho, d^{\prime}}} \frac{1}{\mu\left(B^{\prime}\right)} \int_{B^{\prime}} \exp \left(\frac{C_{3}}{\|f\|_{*, \rho, d^{\prime}}}\left|f-\langle f\rangle_{B^{\prime}}\right|\right) d \mu \leq C_{4}^{2} .
$$


Indeed, denoting $A:=\frac{C_{3}^{\prime}}{\|f\|_{*, \rho, d^{\prime}}}$, for any $c \in \mathbb{C}$ and $B^{\prime} \in \mathcal{O}_{\rho, d^{\prime}}$ we write

$$
\begin{aligned}
& \frac{1}{\mu\left(B^{\prime}\right)} \int_{B^{\prime}} \exp \left(A\left|f-\langle f\rangle_{B^{\prime}}\right|\right) d \mu \\
& \quad \leq \exp \left(A\left|c-\langle f\rangle_{B^{\prime}}\right|\right) \frac{1}{\mu\left(B^{\prime}\right)} \int_{B^{\prime}} \exp (A|f-c|) d \mu \\
& \quad \leq\left(\frac{1}{\mu\left(B^{\prime}\right)} \int_{B^{\prime}} \exp (A|f-c|) d \mu\right)^{2},
\end{aligned}
$$

the last inequality being implied by Jensen's inequality. Therefore (3.5) follows by taking the infimum over $c \in \mathbb{C}$ on the right hand side.

In the following example we comment on possible relations between local $B M O$ spaces for different but equivalent locality functions or metrics.

Example 3.6. Take $X=\mathbb{N}$ with the usual distance $d$ and the counting measure $\mu$. Let $\rho$ be defined by: $\rho(1)=2, \rho(n)=1$ for $n \geq 2$. Then $L_{\text {loc, }, d, d}^{1}(\mathbb{N})=s(\mathbb{N})=B M O_{\rho, d}(\mathbb{N})$ (note that $\|f\|_{*, \rho, d}>0$ for some $\left.f \in B M O_{\rho, d}(\mathbb{N})\right)$. It is clear that for the equivalent locality function $\rho^{\prime}:=2 \rho$ one has $L_{\text {loc }, \rho^{\prime}, d}^{1}(\mathbb{N})=s(\mathbb{N})=L_{\text {loc }, \rho, d}^{1}(\mathbb{N})$. But $B M O_{\rho^{\prime}, d}(\mathbb{N}) \neq$ $B M O_{\rho, d}(\mathbb{N})$ because $f$ defined by $f\left(k^{2}\right)=k, f\left(k^{2}+1\right)=-k$ and 0 otherwise, does not belong to $B M O_{\rho^{\prime}, d}(\mathbb{N})$. The same effect happens when we keep the locality function $\rho$ fixed but change the metric onto an equivalent one, $d^{\prime}:=\frac{1}{2} d$; clearly then $L_{\text {loc }, \rho, d^{\prime}}^{1}(\mathbb{N})=L_{\text {loc }, \rho, d}^{1}(\mathbb{N})$ but $B M O_{\rho, d^{\prime}}(\mathbb{N}) \neq B M O_{\rho, d}(\mathbb{N})$ (note that $B M O_{\rho, d^{\prime}}(\mathbb{N})=B M O_{\rho^{\prime}, d}(\mathbb{N})$ ).

Finally we furnish a simple example of a measure metric space where the John-Nirenberg inequality fails to hold.

Example 3.7. Take $X=\mathbb{N}$ with the $0-1$ metric and the measure $\mu$ given by $\mu(\{n\})=\frac{1}{3} 4^{-n}$; recall that $\mu$ is not even local doubling. Then $L_{\text {loc }}^{1}(\mathbb{N})=\ell^{1}(\mathbb{N}, \mu)=B M O(\mathbb{N}, \mu)$. Since the mean oscillation of any $f=\{f(n)\} \in \ell^{1}(\mathbb{N}, \mu)$ over every one-element ball equals 0 , therefore $\|f\|_{*}$ is just the mean oscillation over $B=\mathbb{N}$ and the John-Nirenberg inequality refers to this single ball as well. The inequality

$$
\mu\left(\left\{n \in \mathbb{N}:\left|f(n)-\langle f\rangle_{\mathbb{N}}\right|>\lambda\right\}\right) \leq C_{1} \exp \left(-C_{2} \lambda /\|f\|_{*}\right), \quad \lambda>0,
$$

cannot hold for $f \in B M O(\mathbb{N}, \mu)$, because taking $f=\left\{2^{n}\right\} \in \ell^{1}(\mathbb{N}, \mu)$ one notes that for $\lambda_{N}=2^{N}, N \in \mathbb{N}$, the left hand side equals $\frac{1}{9} 4^{-N}$ and the right hand side, $\exp \left(-C_{2} 2^{N} /\|f\|_{*}\right)$, decays much faster with $N \rightarrow \infty$. 


\section{Local $A_{p}$-weights}

This section deals with the local $A_{p}$-weights. We examine basic properties of them and prove a result on weighted $L^{p}$ boundedness of the maximal operator $M_{\rho}$. An important question usually asked for maximal operators is that about weighted norm inequalities. A fundamental result of Muckenhoupt states that the Hardy-Littlewood maximal operator $M$ on $\mathbb{R}^{n}$ is bounded on $L^{p}\left(\mathbb{R}^{n}, w d x\right), 1<p<\infty$, or bounded from $L^{1}\left(\mathbb{R}^{n}, w d x\right)$ into $L^{1, \infty}\left(\mathbb{R}^{n}, w d x\right), p=1$, if and only if $w$ satisfies the so called $A_{p}$-condition. There is an abundance of articles where Muckenhoupt's result was generalized in different directions. In particular, it was extended into the setting of measure metric spaces with doubling property. Orobitg and Pérez [17] further extended the theory to nondoubling measures in $\mathbb{R}^{n}$.

The concept of $A_{p}$ weights carries over onto measure metric spaces. In this section we focus on developing, in reasonable limits, a theory of local $A_{p}$ weights. By a weight on $(X, \mu)$ we mean a Borel measurable function $w: X \rightarrow[0, \infty]$. For a weight $w$ we shall consider the Borel measure $w d \mu$ and use the same symbol $w$ to denote this measure; thus for a Borel set $E \subset X$ we shall write $w(E)=\int_{E} w d \mu$. Given a system $(X, d, \mu, \rho)$ and $1 \leq p<\infty$, a weight $w$ is said to satisfy the $A_{p, \rho}$-condition provided

$$
\left(\int_{B} w d \mu\right)^{1 / p}\left(\int_{B} w^{-p^{\prime} / p} d \mu\right)^{1 / p^{\prime}} \leq C \mu(B), \quad B \in \mathcal{O}_{\rho},
$$

where $p^{\prime}$ denotes the conjugate exponent, $\frac{1}{p}+\frac{1}{p^{\prime}}=1$; if $p=1$, then (4.1) is understood as

$$
\int_{B} w d \mu \cdot \underset{x \in B}{\operatorname{esssup}} \frac{1}{w(x)} \leq C \mu(B), \quad B \in \mathcal{O}_{\rho} .
$$

Here, by convention, $0 \cdot \infty=0$.

The class of weights satisfying (4.1) will be denoted as $A_{p, \rho}=$ $A_{p, \rho}(X, d, \mu)$. If $\rho$ is a locality function, then we will refer to (4.1) as to the local $A_{p}$-condition and to $w \in A_{p, \rho}$ as to a local $A_{p}$-weight. If $\rho \equiv \infty$, then (4.1) is the usual $A_{p}$-condition for the measure metric space $(X, d, \mu)$ and we write $A_{p}(X)$ in place of $A_{p, \infty}(X)$. Local $A_{p, \rho}$ weights in the context of $\mathbb{R}^{n}$ with Lebesgue measure, $d=d_{\infty}$ and $\rho \equiv$ constant, were considered by Rychkov $[\mathbf{1 9}]$.

A number of basic facts concerning $A_{p}$ weights holds in the context of any basis, i.e. a distinguished family of open subsets of a measure metric space, see $[\mathbf{1 1}]$. In particular, choosing $\mathcal{O}_{\rho}$ as a basis we have: 
- for $1 \leq p<q$, one has $A_{p, \rho} \subset A_{q, \rho}$;

- for $1<p<\infty, w \in A_{p, \rho}$ if and only if $w^{1-p^{\prime}} \in A_{p^{\prime}, \rho}$;

- for $1<p<\infty$, if $w_{1}, w_{2} \in A_{1, \rho}$, then $w_{1} w_{2}^{1-p} \in A_{p, \rho}$.

The following lemma clarifies the picture of attaining the extreme values 0 and $\infty$ by an $A_{p, \rho}$ weight.

Lemma 4.1. Let $1 \leq p<\infty$ and $w \in A_{p, \rho}$. Then, on every $\rho$-component $U$ of $X$, one of the following three possibilities holds:

(1) $w(x)=0$, $\mu$-a.e. $x \in U$;

(2) $w(x)=\infty$, $\mu$-a.e. $x \in U$;

(3) $0<w(x)<\infty$, $\mu$-a.e. $x \in U$.

Proof: Let $U, 1 \leq p<\infty$ and $w \in A_{p, \rho}$ be fixed. It is easily seen that (4.1) implies the following: for every $B \in \mathcal{O}_{\rho}$, if $w(B)>0$, then $w(x)>$ $0, \mu$-a.e. $x \in B$. Assume that the possibility (1) does not hold. This implies that there exists a ball $B_{0} \in \mathcal{O}_{\rho}, B_{0} \subset U$, such that $w\left(B_{0}\right)>0$ and hence $w(x)>0, \mu$-a.e. $x \in B_{0}$. Let $x_{0}$, the center of $B_{0}$, be the reference point for $U$. Given any other point $y \in U$ let $\left\{B_{1}, \ldots, B_{m}\right\} \subset$ $\mathcal{O}_{\rho}$ be a sequence of balls such that $x_{0} \in B_{1}, y \in B_{m}$ and $B_{i} \cap B_{i+1} \neq \varnothing$, $i=1, \ldots, m-1$. Since $\mu\left(B_{0} \cap B_{1}\right)>0$ and $w(x)>0, \mu$-a.e. $x \in B_{0} \cap B_{1}$, we have $w\left(B_{1}\right)>0$. Repeating the argument several times we conclude that $w(x)>0, \mu$-a.e. $x \in B_{m}$. Thus we showed that for every $y \in U$ there is a ball $B_{y} \in \mathcal{O}_{\rho}$ containing $y$ and such that $w(x)>0, \mu$-a.e. $x \in B_{y}$. This implies that $w(x)>0, \mu$-a.e. $x \in U$. Assuming in addition that also the possibility (2) does not hold, we essentially repeat the argument for $w^{-p^{\prime} / p}$ (or $w^{-1}$ when $p=1$ ) in place of $w$ to conclude that $w(x)<\infty, \mu$-a.e. $x \in U$. This finishes the proof of Lemma 4.1.

In what follows we exclude from our consideration 'extreme' $A_{p^{-}}$ weights, i.e. those weights which equal 0 or $\infty \mu$-a.e. on a $\rho$-component of $X$. Thus, from now on, given a weight $w$ we assume that $0<w(x)<$ $\infty, \mu$-a.e. $x \in X$. This additional assumption imposed on $w$ satisfying (4.1), together with the agreement $0 \cdot \infty=0$, easily implies that such a $w$ has the properties: $w \in L_{\mathrm{loc}, \rho}^{1}(X)$ and $w^{-1 / p} \in L_{\mathrm{loc}, \rho}^{p^{\prime}}(X)$; consequently, $w d \mu$ is a Borel measure on $X$ such that $0<w(B)<\infty$ for any ball $B$. (Note, however, that in general $w d \mu$ is not necessarily Radon when $\mu$ is.)

Given a general weight $w \in L_{\text {loc, } \rho}^{1}(X)$ such that $w(B)>0$ for every ball $B \in \mathcal{O}_{\rho}$, define

$$
M_{\rho, w} f(x)=\sup _{x \in B \in \mathcal{O}_{\rho}} \frac{1}{w(B)} \int_{B}|f| w d \mu,
$$


and for $1 \leq p<\infty$, let $L_{w}^{p}(X)=L^{p}(X, w d \mu)$ denote the weighted Lebesgue $L^{p}$ space, i.e. the $L^{p}$ space with respect to $w d \mu$.

Jawerth $[\mathbf{1 1}]$ gave a different proof of celebrated Muckenhoupt's theorem as a corollary to a much more general result. Though Jawerth's theorem was proved in the Euclidean setting of $\mathbb{R}^{n}$ with Lebesgue measure and a general basis, an elegant proof of this theorem delivered by Lerner [12] shows that the theorem is valid also in the context of measure metric spaces and arbitrary basis. Specified to $\mathcal{O}_{\rho}$ as a basis it says the following.

Theorem 4.2 (Jawerth, $[\mathbf{1 1}])$. Given $(X, d, \mu, \rho)$ and $1<p<\infty$, let $w$ be a weight and $\sigma=w^{1-p^{\prime}}$. Then the following are equivalent:

(1) $M_{\rho}$ is bounded on both $L_{w}^{p}(X)$ and $L_{\sigma}^{p^{\prime}}(X)$;

(2) $w \in A_{p, \rho}, M_{\rho, w}$ is bounded on $L_{w}^{p^{\prime}}(X)$ and $M_{\rho, \sigma}$ is bounded on $L_{\sigma}^{p}(X)$.

As a corollary to Theorem 4.2 we obtain the following.

Proposition 4.3. Let $\mu$ satisfy the $\rho$-local 5-condition, $1<p<\infty$, w be a weight, $A_{p, \rho}=A_{p, 5 \rho}$ and $A_{p^{\prime}, \rho}=A_{p^{\prime}, 5 \rho}$. Then $M_{\rho}: L_{w}^{p}(X) \rightarrow L_{w}^{p}(X)$ boundedly if and only if $w \in A_{p, \rho}$. Moreover, the same statement is valid with 5 is replaced by 3 , when $\mu$ is Radon and $w$ is such that $w d \mu$ is also Radon.

Proof: The fact that if $M_{\rho}: L_{w}^{p}(X) \rightarrow L_{w}^{p}(X)$ boundedly, then $w \in A_{p, \rho}$, is standard; see e.g. [6, Chapter 7], where the argument delivered in the Euclidean setting may be readily adapted to a general context of measure metric spaces with arbitrary basis. To prove the converse take $w \in A_{p, \rho}$ and note that then $\sigma \in A_{p^{\prime}, \rho}$, and hence to apply Theorem 4.2 we have only to check that $M_{\rho, w}$ is bounded on $L_{w}^{p^{\prime}}(X)$ (boundedness of $M_{\rho, \sigma}$ on $L_{\sigma}^{p}(X)$ goes analogously). This task reduces to verifying that $w d \mu$ satisfies the $\rho$-local 5 -condition provided $\mu$ does; then Proposition 2.2 and interpolation give the required bound of $M_{\rho, w}$ on $L_{w}^{p^{\prime}}(X)$. We shall prove the latter claim by using a standard argument and the assumption made on the $A_{p, \rho}$ class. Indeed, take $B \in \mathcal{O}_{\rho}$ so that $5 B \in \mathcal{O}_{5 \rho}$. Since $w \in A_{p, 5 \rho}$, (4.1) is applicable to $5 B$, and hence, by Hölder's inequality,

$$
\begin{aligned}
\mu(B)^{p} w(5 B) & =\left(\int_{5 B} \chi_{B} d \mu\right)^{p} w(5 B) \leq w(B)\left(\int_{5 B} w^{-p^{\prime} / p} d \mu\right)^{p / p^{\prime}} w(5 B) \\
& \leq C w(B) \mu(5 B)^{p}
\end{aligned}
$$

and $w(5 B) \leq C^{\prime} w(B)$ follows. The claim is proved and the proof of the proposition is finished. 
It is obvious that $\rho^{\prime} \leq \rho$ implies $A_{p, \rho} \subset A_{p, \rho^{\prime}}$. Examples show that for a locality function $\rho^{\prime}$ the inclusion $A_{p} \subset A_{p, \rho^{\prime}}$ may be proper, see [13]. On the other hand, if $\rho^{\prime} \simeq \rho$, there is no reason to expect that in general, $A_{p, \rho}=A_{p, \rho^{\prime}}$. However, in the particular case of $\left(\Omega_{0}, d_{\infty}, d x\right)$, see Section 5 for the definition, it was shown in [13, Proposition 2.2] that $A_{p, k}=A_{p, l}, 1 \leq p<\infty, 0<k, l<1$, for the equivalent locality functions $\rho_{k}$ and $\rho_{l}$. In particular, for $0<k<1 / 3$ it holds $A_{p, k}=A_{p, 3 k}$, and hence for this range of $k$ and $1<p<\infty$, by Proposition 4.3 the conclusion of [13, Theorem 4.1] follows, i.e. $M_{k}$ is bounded on $L_{w}^{p}\left(\Omega_{0}, d x\right)$ provided $w \in A_{p, k}$. Let us point out, however, that the main achievement of [13, Theorem 4.1] is included in the weak type $(1,1)$ result.

Example 4.4. Take $X=\mathbb{N}$ and $\mu, d, d^{\prime}, \rho, \rho^{\prime}$ as in Remarks 2.4 and 2.5 and let $1 \leq p<\infty$ be given. Then $A_{p, \rho}=A_{p, \rho}(\mathbb{N}, d, \mu)$ consists of all sequences $\{w(n)\}_{1}^{\infty}$ such that $0 \leq w(n) \leq \infty$, while $A_{p, \rho^{\prime}}=A_{p, \rho^{\prime}}(\mathbb{N}, d, \mu)$ is the set of all sequences $\{w(n)\}_{1}^{\infty}$ such that $0<w(n)<\infty$, and in addition satisfying $\left(\sum w(n) a_{n}\right)^{1 / p}\left(\sum w(n)^{-p^{\prime} / p} a_{n}\right)^{1 / p^{\prime}}<\infty$ (recall that $\left.\mu(\{n\})=a_{n}\right)$. Thus $A_{p, \rho^{\prime}}$ is a proper subset of $A_{p, \rho}$ though $\rho^{\prime} \simeq \rho$. For similar reasons $A_{p, \rho, d^{\prime}}$ is a proper subset of $A_{p, \rho, d}$ though $d^{\prime} \simeq d$.

\section{Locality function of Whitney type}

In this section we focus on studying objects associated to locality function of Whitney type. This was the subject investigated earlier in [13], [14]. Let $(X, d, \mu)$ be fixed. Assume $\Omega$ is an open and nontrivial subset of $X$, and let $\partial \Omega \neq \varnothing$ be its boundary. For any fixed scale parameter $k \in(0,1)$ we define the locality function $\rho_{k}: \Omega \rightarrow(0, \infty)$ by

$$
\rho_{k}(x)=k \cdot d(x, \partial \Omega), \quad x \in \Omega,
$$

where the distance of $x \in \Omega$ to $\partial \Omega$ is

$$
d(x, \partial \Omega):=\inf \{d(x, y): y \in \partial \Omega\} .
$$

Clearly $\rho_{k}$ is a l.s.c. function hence all relevant considerations of previous sections apply. To be precise the measure metric space we consider from now on is $(\Omega, d, \mu)$, where, for the sake of simplicity, we use the same characters $d$ and $\mu$ to denote the restrictions to $\Omega$ of the distance and measure originally defined on $X$. We shorten the notation writing $M_{k}$ rather than $M_{\rho_{k}}$; similar remark applies in the whole context and we write $\mathcal{O}_{k}, L_{\text {loc }, k}^{1}(\Omega), A_{k}^{p}(\Omega)$, etc. It is important to point out here that if $B=B(x, r) \in \mathcal{O}_{k}$ and $x \in \Omega$, then $B \subset \Omega$.

If $X=\mathbb{R}^{n}$ and $\Omega$ is its open nontrivial subset, then for the equivalent locality functions $\rho_{k}$ and $\rho_{l}, 0<k, l<1$, we have $L_{\text {loc }, k}^{1}(\Omega)=L_{\text {loc }, l}^{1}(\Omega)$. 
This is because the covering property stated in Remark 2.6 holds for the pair $\mathcal{O}_{k}$ and $\mathcal{O}_{l}$. This space, independent of $k \in(0,1)$, will be denoted by $L_{\text {loc,* }}^{1}(\Omega)$. Similarly, given $0<k<1$, for the equivalent metrics $d_{2}$ and $d_{\infty}$ we have $L_{\text {loc }, k, d_{2}}^{1}(\Omega)=L_{\text {loc }, k, d_{\infty}}^{1}(\Omega)$. This time this is justified by the fact that the covering property stated in Remark 2.7 holds for the pair $\mathcal{O}_{k, d_{2}}$ and $\mathcal{O}_{k, d_{\infty}}$.

It is worth mentioning that the limiting case $k=1$, though qualitatively different from that of $k \in(0,1)$, also falls within the scope of just presented theory. Let us illustrate this by considering the issue of choosing proper definitions of the Hardy-Littlewood maximal operator and the $B M O$ space on a general open proper subset $\Omega$ of $\mathbb{R}^{n}$ equipped with Lebesgue measure. There are several ways of such definitions. For the Hardy-Littlewood maximal operator one way, a tempting one, is to define $M_{\Omega}$ as a maximal operator on the measure metric space $\Omega$ with Euclidean distance and measure inherited from $\mathbb{R}^{n}$,

$$
M_{\Omega} f(x)=\sup _{x \in B} \frac{1}{|B \cap \Omega|} \int_{B \cap \Omega}|f|, \quad x \in \Omega ;
$$

here $B$ is a Euclidean ball so that $B \cap \Omega$ is a ball related to $\Omega$ and $|B \cap \Omega|$ means the Lebesgue measure of $B \cap \Omega$. But then the essential trouble lies in the fact that the doubling property is not stable under restrictions to subsets, so, in general, the weak type $(1,1)$ inequality cannot be proved by using usual arguments. Another way (in some sense a "right" one) is to define $M_{\Omega}$ as

$$
M_{\Omega} f(x)=\sup _{x \in B \subset \Omega} \frac{1}{|B|} \int_{B}|f|, \quad x \in \Omega
$$

where the supremum is taken over all balls that contain $x$ and are entirely contained in $\Omega$; see, for instance, [7, Section 4]. Note that for $M_{\Omega}$ defined by (5.1) we have $M_{\Omega}=M_{\rho_{1}}$ so that the definition has a local flavour. Hence we can apply Proposition 2.2 to check that $M_{\Omega}$ is of weak type $(1,1)$; indeed, Lebesgue measure on $\Omega$ satisfies the $\rho_{1}$-local 3-condition.

Similar indecisions accompany the definition of $B M O(\Omega)$. One can use the Euclidean balls related to $\Omega$, but again the "right" way seems to be the following: $B M O(\Omega)$ is defined as the space of these functions $f \in L_{\text {loc }}^{1}(\Omega)$ (the class of functions integrable on any ball $B \subset \Omega$ ) such that

$$
\sup _{B \subset \Omega} \frac{1}{|B|} \int_{B}\left|f-\langle f\rangle_{B}\right|, \quad x \in \Omega
$$


where the supremum is taken over all balls entirely contained in $\Omega$; see [7, Remark 2.3]. Note that then the definition again has a local flavor since $B M O(\Omega)$ defined in this way coincides with $B M O_{\rho_{1}}(\Omega)$.

We now specify our considerations to the case $\Omega_{0}=\mathbb{R}^{n} \backslash\{0\}$ equipped with Lebesgue measure and the distance $d$ being either $d_{\infty}$ or $d_{2}$ (induced by the norm $\|\cdot\|$ being either $\|\cdot\|_{\infty}$ or $\|\cdot\|_{2}$, respectively). Thus the locality function $\rho_{k}, k \in(0,1)$, is given by

$$
\rho_{k}(x)=k\|x\|, \quad x \in \Omega_{0} .
$$

Since $\Omega_{0}$ differs from $\mathbb{R}^{n}$ by a single point, which is a set of Lebesgue measure zero, therefore the relevant objects on $\Omega_{0}$ and $\mathbb{R}^{n}$ may be identified. Consequently, we can try to compare the properties of $M_{k}^{c}$ and $M_{k}$ with properties of the centered and non-centered Hardy-Littlewood maximal operators on $\mathbb{R}^{n}$. Note that $L_{\text {loc,* }}^{1}\left(\Omega_{0}\right)$ coincides with the space of functions on $\Omega_{0}$ satisfying $\int_{\{a \leq\|x\| \leq b\}}|f|<\infty$ for every $0<a<b<\infty$.

We start with the following observation: if $\operatorname{supp} f \subset\left\{x \in \mathbb{R}^{n}: a \leq\right.$ $\|x\| \leq b\}$, where $0<a<b \leq \infty$, then

$$
\operatorname{supp} M_{k}^{c} f \subset\left\{\frac{1}{1+k} a \leq\|x\| \leq \frac{1}{1-k} b\right\}
$$

and

$$
\operatorname{supp} M_{k} f \subset\left\{\frac{1-k}{1+k} a \leq\|x\| \leq \frac{1+k}{1-k} b\right\} .
$$

It is well known, cf. [3, p. 117], that $M f \notin L^{1}\left(\mathbb{R}^{n}\right)$ whenever $f \in L^{1}\left(\mathbb{R}^{n}\right)$ and $f \neq 0$. In the local case, however, taking $f$ bounded and with bounded support, also $M_{k} f$ is bounded and with bounded support, hence $M_{k} f \in L^{1}\left(\Omega_{0}\right)$. On the other hand, one can easily find an $f \in L^{1}\left(\Omega_{0}\right)$ such that $M_{k}^{c} f \notin L^{1}\left(\Omega_{0}\right)$, even worse, $M_{k}^{c} f \notin L_{\mathrm{loc}, *}^{1}\left(\Omega_{0}\right)$. Indeed, to show this in the case $n=1$ we can use the (shifted) example from $[\mathbf{3}$, p. 118],

$$
f(x)= \begin{cases}\frac{1}{(x-1)(\log (x-1))^{2}}, & 1<x<3 / 2, \\ 0, & x \in \Omega_{0} \backslash(1,3 / 2),\end{cases}
$$

and consider, for simplicity, $k=1 / 2$. Then, for $1<y<5 / 4$, we have $(1,2 y-1) \in \mathcal{O}_{1 / 2}(y)$ and

$$
M_{1 / 2}^{c} f(y) \geq \frac{1}{2(y-1)} \int_{1}^{2 y-1} f(x) d x=\frac{1}{2(y-1)(-\log (2(y-1)))}
$$

hence $\int_{1}^{5 / 4} M_{1 / 2}^{c} f(y) d y=\infty$. 
It was discovered in [2] that the following dichotomy holds: for any function $f \in B M O\left(\mathbb{R}^{n}\right)$ either $M f \equiv \infty$ or $M f<\infty, x$-a.e. Later on it was observed that the property

"if $M f\left(x_{0}\right)<\infty$ for some $x_{0} \in \mathbb{R}^{n}$, then $M f(x)<\infty$, x-a.e."

should be linked to the bigger class $L_{\text {loc }}^{1}\left(\mathbb{R}^{n}\right)$ rather than only to $B M O\left(\mathbb{R}^{n}\right)$, see [7, Theorem 2.2] and remarks following this theorem. In $[\mathbf{1}]$ an elegant argument was furnished to show that the above-mentioned property holds in the general setting of measure metric spaces with doubling measures.

Here we show that the dichotomy we mentioned above does not take place in the local context.

Proposition 5.1. Let $f \in L_{\mathrm{loc}, *}^{1}\left(\Omega_{0}\right)$. Then $M_{k} f(x)<\infty$, $\mu$-a.e. $x \in X$.

Proof: We start with observing that if $\operatorname{supp} g \subset\{0<\|x\| \leq a\} \cup\{b \leq$ $\|x\| \leq \infty\}$, where $g \in L_{\mathrm{loc}, *}^{1}\left(\Omega_{0}\right)$ and $0<a<b<\infty$, then

$$
\operatorname{supp} M_{k} g \subset\left\{0<\|x\| \leq \frac{1+k}{1-k} a\right\} \cup\left\{\frac{1-k}{1+k} b \leq\|x\| \leq \infty\right\} .
$$

Now, for any $n \in \mathbb{N}$ satisfying $n^{2}>\frac{1+k}{1-k}$, let

$$
f_{n}=f \chi_{\left\{(1-k) \frac{1}{n} \leq\|x\| \leq(1+k) n\right\}} .
$$

By sublinearity of $M_{k}$,

$$
M_{k} f(x) \leq M_{k} f_{n}(x)+M_{k}\left(f-f_{n}\right)(x)
$$

for every $x \in \Omega_{0}$. Since $f_{n} \in L^{1}\left(\Omega_{0}\right)$, we have $M_{k} f_{n}(x)<\infty$, $\mu$-a.e. $x \in X$, by the weak type $(1,1)$ estimate satisfied by $M_{k}$. But $M_{k}(f-$ $\left.f_{n}\right)(x)=0$ for every $x$ from the region $\frac{1}{n} \leq\|x\| \leq n$, hence also $M_{k} f$ is finite $\mu$-a.e. in this region. Letting $n \rightarrow \infty$ finishes the proof.

Finally we furnish examples that show that in general $M_{\rho}$ and $M_{\rho}^{c}$ are not comparable and the same happens with either $M_{\rho}$ and $M_{\rho^{\prime}}$ for $\rho \simeq \rho^{\prime}$, or $M_{\rho, d}$ and $M_{\rho, d^{\prime}}$ for $d \simeq d^{\prime}$.

Example 5.2. For $f_{0}=\chi_{\{1 \leq\|x\| \leq 2\}}$ it is easily seen that $\operatorname{supp}^{o} M_{k}^{c} f_{0}=$ $\left\{\frac{1}{1+k}<\|x\|<\frac{2}{1-k}\right\}$ and $\operatorname{supp}^{o} M_{k} f_{0}=\left\{\frac{1-k}{1+k}<\|x\|<2 \frac{1+k}{1-k}\right\}$. Thus $M_{k} f_{0} \lesssim M_{k}^{c} f_{0}$ fails, hence $M_{k} f \simeq M_{k}^{c} f$ cannot hold, uniformly in $f \in$ $L_{\text {loc, },}^{1}\left(\Omega_{0}\right)$.

Example 5.3. Fix $0<k<l<1$. For $f_{0}$ as in Example 5.2, by comparing supp ${ }^{o} M_{k} f_{0}$ with $\operatorname{supp}^{o} M_{l} f_{0}$, it is clear that $M_{l} f_{0} \lesssim M_{k} f_{0}$ fails, hence $M_{k} f \simeq M_{l} f$ cannot hold, uniformly in $f \in L_{\text {loc }, c}^{1}\left(\Omega_{0}\right)$, though $\rho_{k}$ 
and $\rho_{l}$ are equivalent. Analogous remark applies to the pair of centered maximal operators, $M_{k}^{c}$ and $M_{l}^{c}$.

Example 5.4. This example aims to show that for $n \geq 2$, the relation $M_{k, d_{\infty}}^{c} f \simeq M_{k, d_{2}}^{c} f$ cannot hold uniformly in $f \in L_{\text {loc }, c}^{1}\left(\Omega_{0}\right)$, though the metrics $d_{\infty}$ and $d_{2}$ are equivalent (again, we use an additional subscript to indicate the dependence of the local maximal operator on a metric). In fact we show something more, namely neither $M_{k, d_{\infty}}^{c} f \lesssim M_{k, d_{2}}^{c} f$ nor $M_{k, d_{2}}^{c} f \lesssim M_{k, d_{\infty}}^{c} f$ holds. For simplicity, consider the case $n=2$ only. Indeed, a calculation shows that the segment

$$
\left\{(t, 2): \frac{2}{1-k}<t<\frac{2\left(1+k \sqrt{2-k^{2}}\right)}{1-k^{2}}\right\}
$$

belongs to $\operatorname{supp}^{o} M_{k, d_{2}}^{c} f_{0}$, but clearly is outside $\operatorname{supp}^{o} M_{k, d_{\infty}}^{c} f_{0}$; here $f_{0}$ is as in Example 5.2 with the norm $\|\cdot\|$ specified to $\|\cdot\|_{\infty}$. Consequently, $M_{k, d_{2}}^{c} f_{0} \lesssim M_{k, d_{\infty}}^{c} f_{0}$ cannot hold. On the other hand, for $g_{0}=\chi_{\left\{1 \leq\|x\|_{2} \leq 2\right\}}$ we have $\operatorname{supp}^{o} M_{k, d_{2}}^{c} g_{0}=\left\{\frac{1}{1+k}<\|x\|_{2}<\frac{2}{1-k}\right\}$, which occurs to be properly contained in $\operatorname{supp}^{o} M_{k, d_{\infty}}^{c} g_{0}$, hence $M_{k, d_{\infty}}^{c} g_{0} \lesssim$ $M_{k, d_{2}}^{c} g_{0}$ cannot hold either.

In spite of the above (negative) examples it is probably worth pointing out that in the case of $\Omega_{0}$, there are some relations between maximal operators associated to different metrics. Namely we have

$$
M_{k, d_{\infty}}^{c} f \lesssim M_{k \sqrt{n}, d_{2}}^{c} f, \quad M_{k, d_{2}}^{c} f \lesssim M_{k \sqrt{n}, d_{\infty}}^{c} f, \quad f \in L_{\mathrm{loc}, c}^{1}\left(\Omega_{0}\right) ;
$$

for this, we can invoke an argument from the proof of [14, Theorem 4.2].

\section{References}

[1] D. Aalto And J. Kinnunen, The discrete maximal operator in metric spaces, J. Anal. Math. 111 (2010), 369-390. DOI: 10.1007/ s11854-010-0022-3.

[2] C. Bennett, R. A. DeVore, and R. Sharpley, Weak- $L^{\infty}$ and BMO, Ann. of Math. (2) 113(3) (1981), 601-611. DOI: 10.2307/ 2006999.

[3] C. Bennett and R. Sharpley, "Interpolation of operators", Pure and Applied Mathematics 129, Academic Press, Inc., Boston, MA, 1988.

[4] A. Carbonaro, G. Mauceri, and S. Meda, $H^{1}$ and BMO for certain locally doubling metric measure spaces, Ann. Sc. Norm. Super. Pisa Cl. Sci. (5) 8(3) (2009), 543-582. 
[5] A. Carbonaro, G. Mauceri, and S. Meda, $H^{1}$ and BMO for certain locally doubling metric measure spaces of finite measure, Colloq. Math. 118(1) (2010), 13-41. DOI: 10.4064/cm118-1-2.

[6] J. Duonndikoetxea, "Fourier analysis", Translated and revised from the 1995 Spanish original by David Cruz-Uribe, Graduate Studies in Mathematics 29, American Mathematical Society, Providence, RI, 2001.

[7] A. Fiorenza and M. Krbec, On the domain and range of the maximal operator, Nagoya Math. J. 158 (2000), 43-61.

[8] G. B. Folland, "Real analysis". Modern techniques and their applications, Second edition, Pure and Applied Mathematics (New York), A Wiley-Interscience Publication, John Wiley \& Sons, Inc., New York, 1999.

[9] J. Heinonen, "Lectures on analysis on metric spaces", Universitext, Springer-Verlag, New York, 2001. DOI : 10.1007/978-1-46130131-8.

[10] A. D. Ionescu, Fourier integral operators on noncompact symmetric spaces of real rank one, J. Funct. Anal. 174(2) (2000), 274-300. DOI: $10.1006 / \mathrm{jfan} .2000 .3572$.

[11] B. JAwERTh, Weighted inequalities for maximal operators: linearization, localization and factorization, Amer. J. Math. 108(2) (1986), 361-414. DOI: $10.2307 / 2374677$.

[12] A. K. LERner, An elementary approach to several results on the Hardy-Littlewood maximal operator, Proc. Amer. Math. Soc. 136(8) (2008), 2829-2833. DOI: 10.1090/S0002-9939-08-09318-0.

[13] C.-C. Lin And K. Stempak, Local Hardy-Littlewood maximal operator, Math. Ann. 348(4) (2010), 797-813. DOI: 10.1007/s00208010-0499-1.

[14] C.-C. Lin and K. Stempak, $H^{1}$ and $B M O$ spaces of Whitney type, Preprint (2011).

[15] J. Mateu, P. Mattila, A. Nicolau, and J. Orobitg, BMO for nondoubling measures, Duke Math. J. 102(3) (2000), 533-565. DOI : $10.1215 / \mathrm{S} 0012-7094-00-10238-4$.

[16] G. Mauceri and S. Meda, $B M O$ and $H^{1}$ for the Ornstein-Uhlenbeck operator, J. Funct. Anal. 252(1) (2007), 278-313. DOI: $10.1016 / \mathrm{j} . \mathrm{jfa} .2007 .06 .017$.

[17] J. Orobitg and C. PÉrez, $A_{p}$ weights for nondoubling measures in $\mathbb{R}^{n}$ and applications, Trans. Amer. Math. Soc. 354(5) (2002), 2013-2033 (electronic). DOI : 10.1090/S0002-9947-02-02922-7. 
[18] H. M. Reimann And T. Rychener, "Funktionen beschränkter mittlerer Oszillation", Lecture Notes in Mathematics 487, SpringerVerlag, Berlin-New York, 1975.

[19] V. S. RYChKOV, Littlewood-Paley theory and function spaces with $A_{p}^{\text {loc }}$ weights, Math. Nachr. 224 (2001), 145-180. DOI : 10.1002/15222616 (200104) 224:1<145: : AID-MANA145>3.3. CD;2-U.

Chin-Cheng Lin and Ya-Shu Wang:

Department of Mathematics

National Central University

Chung-Li, Taiwan 320

Republic of China

E-mail address: clin@math.ncu.edu.tw

E-mail address: wangys@math.ncu.edu.tw

Krzysztof Stempak:

Instytut Matematyki i Informatyki

Politechnika Wrocławska

Wyb. Wyspiańskiego 27

50-370 Wrocław

Poland

E-mail address: Krzysztof.Stempak@pwr.wroc.pl

and

Katedra Matematyki i Zastosowań Informatyki

Politechnika Opolska

ul. Luboszycka 3

45-036 Opole

Poland

Primera versió rebuda el 27 d'abril de 2012,

darrera versió rebuda el 22 de juny de 2012. 